\title{
Genetics of Bone and Muscle Interactions in Humans
}

\author{
Katerina Trajanoska ${ }^{1}$ • Fernando Rivadeneira ${ }^{1}$ • Douglas P. Kiel ${ }^{2,3,4} \cdot$ David Karasik $^{2,5}$
}

Published online: 28 February 2019

(C) The Author(s) 2019

\begin{abstract}
Purpose of Review To summarize the evidence from recent studies on the shared genetics between bone and muscle in humans. Recent Findings Genome-wide association studies (GWAS) have successfully identified a multitude of loci influencing the variability of different bone or muscle parameters, with multiple loci overlapping between the traits. In addition, joint analyses of multiple correlated musculoskeletal traits (i.e., multivariate GWAS) have underscored several genes with possible pleiotropic effects on both bone and muscle including $M E F 2 C$ and $S R E B F 1$. Notably, several of the proposed pleiotropic genes have been validated using human cells or animal models.

Summary It is clear that the study of pleiotropy may provide novel insights into disease pathophysiology potentially leading to the identification of new treatment strategies that simultaneously prevent or treat both osteoporosis and sarcopenia. However, the role of muscle factors (myokines) that stimulate bone metabolism, as well as osteokines that affect muscles, is in its earliest stage of understanding.
\end{abstract}

Keywords Bone $\cdot$ Muscle $\cdot$ Osteoporosis $\cdot$ Sarcopenia $\cdot$ Genome-wide association study (GWAS) $\cdot$ Pleiotropy

\section{Introduction}

Osteoporosis and sarcopenia are common and costly comorbid diseases of aging, and there is an urgent need to prevent and treat both to reduce their associated morbidity and mortality $[1 \bullet \bullet$. A burgeoning body of work shows that they share many common risk factors and biological pathways such as effects of growth hormone and inflammatory cytokines [2,3]. Moreover, the common mesenchymal origin of bone and muscle cells

This article is part of the Topical Collection on Muscle and Bone

Fernando Rivadeneira

f.rivadeneira@erasmusmc.nl

David Karasik

karasik@hsl.harvard.edu

1 Department of Internal Medicine, Erasmus MC, University Medical Center Rotterdam, Rotterdam, Netherlands

2 Hebrew SeniorLife, Institute for Aging Research, Boston, MA, USA

3 Department of Medicine, Beth Israel Deaconess Medical Center and Harvard Medical School, Boston, MA, USA

4 Broad Institute of Harvard and Massachusetts Institute of Technology, Boston, MA, USA

5 Azrieli Faculty of Medicine, Bar-Ilan University, Safed, Israel underpins the tight link between these conditions from the early stages of embryonic development.

Muscle mass and function are important determinants of skeletal growth and bone mass accrual in growing humans. This adaption of bone tissue to loading follows the principles of Frost's mechanostat theory, i.e., bone growth and bone loss are stimulated by the muscle forces/loads acting upon bone surfaces [4]. In addition to their mechanical interaction, bone and muscle are jointly regulated by hormones, and inextricably linked genetically and molecularly [5]. However, the latter interactions are difficult to observe and measure; thus, their roles are less well recognized. The recent rise of new technologies (arising from genetics, molecular biology etc.) has shed new light on the genetic and molecular interplay between bone and muscle; thus, our understanding of these interactions has evolved over time. In the past few years, studies have endeavored to (1) disentangle the intricate molecular mechanisms that lead to osteoporosis and sarcopenia and (2) develop new treatment strategies by pinpointing drug targets common to both conditions.

The aim of this review is to summarize the evidence from current studies on the shared genetics between bone and muscle in humans. Another recent review has addressed this topic in mouse models [6]. 


\section{Genome-Wide Association Study for Bone or Muscle-Related Phenotypes}

Risk factors affecting osteoporosis and sarcopenia have a strong genetic component, with heritability estimates above $60 \%$ [7]. Genome-wide association studies (GWAS) have identified multiple genetic variants influencing the variability of bone mass (Fig. 1). In total, 62 loci [8-12] have been associated with DXAderived bone mineral density (BMD) at either the femoral neck or lumbar spine, while 36 loci [13] have been associated with total body BMD. Notably, these GWASs have highlighted known bone-active pathways, i.e., OPG-RANK-RANKL, WNT, and mesenchymal differentiation, among others [14]. One of the greatest successes in the osteoporosis field was achieved through the discovery of the BMD locus harboring WNT16, a critical regulator of cortical bone thickness [15] and trabecular bone mass [16]. Moreover, with an ever-growing number of genes discovered by GWASs, novel pathways acting on bone have been identified (e.g., oncogenic and melanogenesis pathways). Recently, 518 loci have been associated with ultrasound-derived heel BMD [17, 18], estimated in more than 400,000 participants of the UK Biobank (UKBB) study. Together, these studies have provided new insights into the pathophysiology of osteoporosis, illustrated by the discovery and functional validation of GPC6 and DAAM2. GPC6 may serve as novel drug target for osteoporosis, since it encodes glypican, which is involved in cellular growth control and differentiation. Moreover, GPC6 loss of function leads to increased bone mineral content and developmental skeletal abnormalities. DAAM2 also may be a potential drug target for osteoporosis as it shows effects on bone strength, porosity, and quality in murine models by indirect regulation of the canonical Wnt signaling. DAAM2 was also expressed in human skeletal muscle [19] (Table 1).

In contrast, the fewer number of GWAS of muscle-related phenotype provide less biological insight about the pathways leading to the development of sarcopenia (Fig. 1). To date, only five loci (HSD17B11, VCAN, ADAMTSL3, IRS1, and
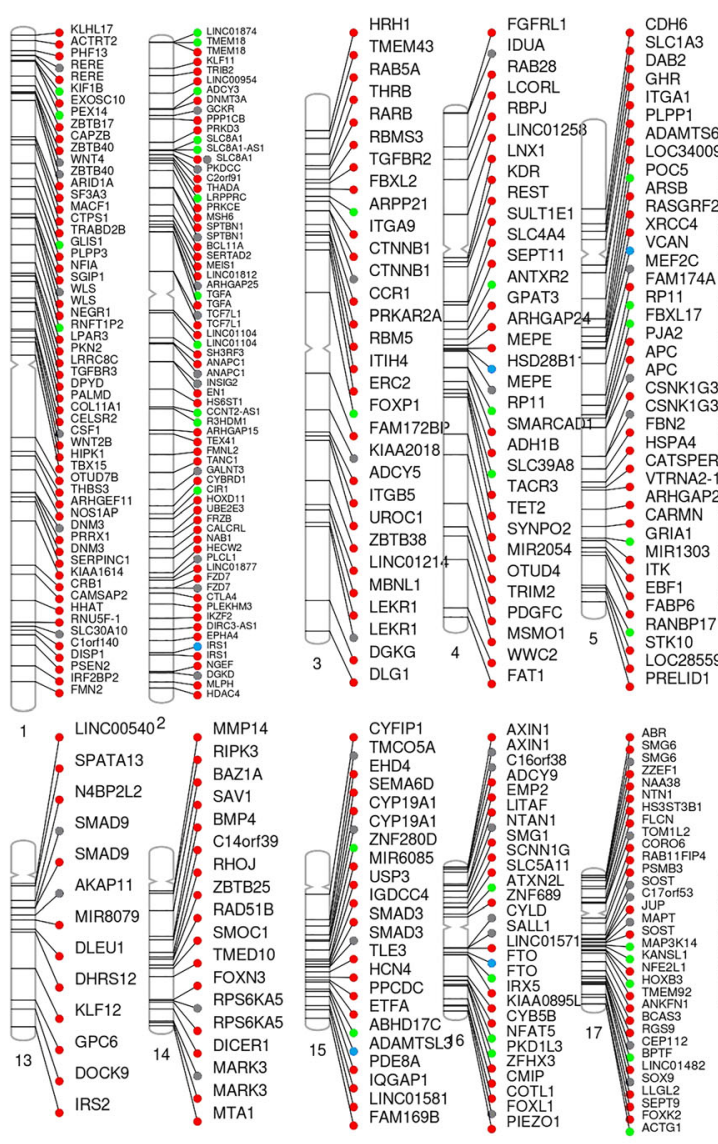

Hand grip

Lean mass

Fig. 1 Phenogram showing genome-wide association study results for bone and muscle-related phenotypes. Genes mapping to loci associated with lean mass (light blue), hand grip (light green), heel ultrasound
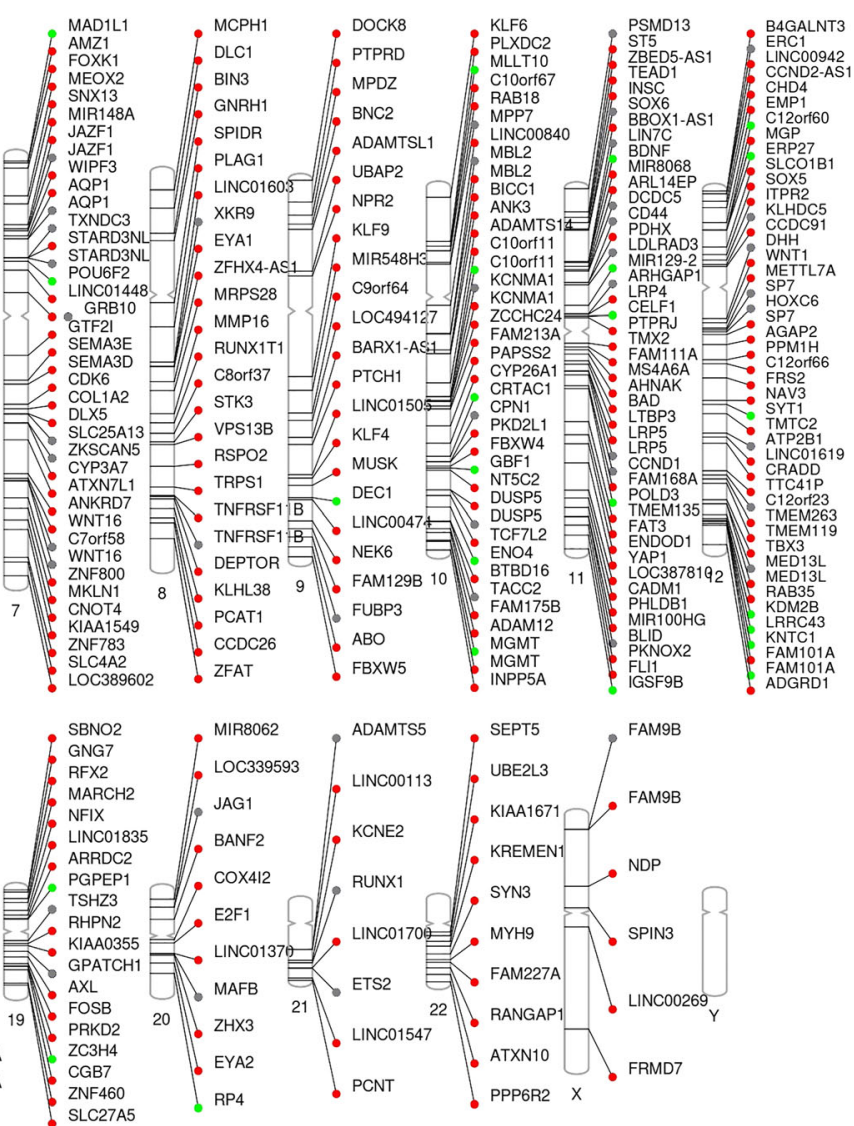

Heel ultrasound eBMD

DXA-BMD

estimated BMD (red), and DXA-derived BMD (gray). The ideogram was constructed using Phenogram http://visualization.ritchielab.psu.edu/ phenograms/plot 
Table 1 Bone genes discovered by UK Biobank (and other GWAS) and evidence of their molecular role in the muscle

\begin{tabular}{lll}
\hline eBMD gene & Muscle-related trait & Reference \\
\hline AHNAK & Gene expression in human skeletal muscle & Su, Ekman et al. 2015 \\
AQP1 & Gene expression in human skeletal muscle & Su, Ekman et al. 2015 \\
ARHGAP26 & Positive/mouse skeletal muscle mass KO & Verbrugge, Schönfelder et al. 2018 \\
BCKDHB & Gene expression in human skeletal muscle & Su, Ekman et al. 2015 \\
DAAM2 & Gene expression in human skeletal muscle & Su, Ekman et al. 2015 \\
DLEU1 & Gene expression in human skeletal muscle & Su, Ekman et al. 2015 \\
GRB10 & Negative/mouse skeletal muscle mass KO & Verbrugge, Schönfelder et al. 2018 \\
HMGA2 & Positive/mouse skeletal muscle mass KO & Verbrugge, Schönfelder et al. 2018 \\
IGFBP2 & Negative/mouse skeletal muscle mass overexpress & Verbrugge, Schönfelder et al. 2018 \\
MMP9 & Positive/mouse skeletal muscle mass overexpress & Verbrugge, Schönfelder et al. 2018 \\
MPP7 & Gene expression in human skeletal muscle & Su, Ekman et al. 2015 \\
PPARD & Positive/mouse skeletal muscle mass overexpress & Verbrugge, Schönfelder et al. 2018 \\
SMAD3 & Positive/ mouse skeletal muscle mass KO & Verbrugge, Schönfelder et al. 2018 \\
SMAD7 & Positive/mouse skeletal muscle mass KO & Verbrugge, Schönfelder et al. 2018 \\
SOX6 & Positive/mouse skeletal muscle mass KO & Verbrugge, Schönfelder et al. 2018 \\
\hline
\end{tabular}

$e B M D$ estimated bone mineral density, $K O$ knock out
FTO) have been robustly associated with lean mass (total and/ or appendicular) [20], which constitutes a good proxy for skeletal muscle mass [21]. Three out of the five lean massassociated SNPs identified by GWAS are significantly enriched in enhancers and promoters acting in muscle cells. Recently, the same study identified TNRV6B as additional lean mass locus after more stringent adjustment for fat [22]. However, the exact biological pathways affecting muscle mass still remain unknown. Two recent grip strength GWAS, a proxy for muscular function, have been more fruitful, yielding 64 muscle strength-related loci [23, 24] identified within the UK Biobank. The loci found associated with grip strength contain genes implicated in the structure and function of skeletal muscle (ACTG1), excitation-contraction coupling (SLC8A1), or involvement in the regulation of neurotransmission ( $S Y T 1$ ), which provides additional evidence of the genetic control exerted on this muscle trait [23]. These findings highlight that the grip strength phenotype has a neuromuscular component, since it also characterizes the ability of the peripheral nervous system to appropriately recruit muscle cells. Briefly, Actg1-ms knockout mice display muscle weakness and whole-body functional deficit [25]. SLC8A1 overexpression in muscle cells induces muscular changes similar to those of muscular dystrophy [26]. Finally, SYT1 has been linked to synaptic defects at the neuromuscular junctions in mouse model of spinal muscular atrophy [27]. Further, three lead SNPs (rs10186876, rs6687430, and rs754512) for grip strength map in the vicinity of genes implicated in monogenic syndromes characterized by neurological and/or psychomotor impairment like French-Canadian variant of Leigh syndrome characterized (LRPPRC), Zellweger Spectrum Peroxisomal
Biogenesis Disorder (PEX14), and Koolen-de Vries syndrome (KANSL1) [23].

\section{From Cross-Phenotype Effects to Pleiotropy: Bone and Muscle}

\section{Basic Concepts}

Multiple genes identified by GWAS of muscle-related traits have also been associated with heel BMD in the UKBB GWAS (Table 2). While such cross-phenotype associations may arise due to biological pleiotropy, there are other reasons that can lead to spurious pleiotropy. Therefore, crossphenotype associations should not be always regarded as the consequence of true pleiotropy. Pleiotropy commonly refers to a phenomenon in which a genetic locus (a gene or a single variant within a gene) affects more than one trait or disease [28]. It can be classified as (1) biological—when a gene has a direct biological effect on more than one trait or biomarker; (2) mediated - where a gene has a biological effect on one trait which lies on the causal path to another trait and thus the gene affects both traits; and (3) spurious - when different forms of biases can lead to false-positive findings [29]. The most common causes of spurious pleiotropy are ascertainment bias and phenotypic misclassification [29]. The study of pleiotropy may have tremendous clinical implications in the fields of osteoporosis and sarcopenia by discovering new drug targets acting on both muscle and bone. 
Table 2 Overlapping genes between different bone parameters and different muscle-related traits

\begin{tabular}{|c|c|c|c|c|}
\hline Muscle-related trait & Gene & eBMD $P$ value & Muscle-related traits $P$ value & Reference \\
\hline \multicolumn{5}{|l|}{ Bone mineral density } \\
\hline Total body lean mass & $M C 4 R$ & $2.0 \times 10^{-15}$ & $1.0 \times 10^{-18}$ & Karasik et al. 2019 \\
\hline Total body lean mass & FTO & $1.6 \times 10^{-26}$ & $1.4 \times 10^{-09}$ & Zillikens et al. 2017 \\
\hline Hand grip strength & IRSI & $4.7 \times 10^{-08}$ & $1.5 \times 10^{-11}$ & Zillikens et al. 2017 \\
\hline Hand grip strength & $M G M T$ & $2.3 \times 10^{-22}$ & $1.0 \times 10^{-13}$ & Tikkanen et al. 2018 \\
\hline Hand grip strength & TCF4 & $9.4 \times 10^{-10}$ & $5.9 \times 10^{-15}$ & Tikkanen et al. 2018 \\
\hline Hand grip strength & TMEM18 & $2.0 \times 10^{-11}$ & $5.4 \times 10^{-22}$ & Tikkanen et al. 2018 \\
\hline Hand grip strength & LINC01104 & $7.9 \times 10^{-11}$ & $3.1 \times 10^{-09}$ & Tikkanen et al. 2018 \\
\hline Hand grip strength & $M C 4 R$ & $2.0 \times 10^{-15}$ & $2.1 \times 10^{-19}$ & Tikkanen et al. 2018 \\
\hline Hand grip strength & PEX14 & $6.7 \times 10^{-13}$ & $5.6 \times 10^{-11}$ & Willems et al. 2017 \\
\hline Hand grip strength & SLC8A1 & $7.4 \times 10^{-38}$ & $7.7 \times 10^{-09}$ & Willems et al. 2017 \\
\hline Hand grip strength & $T G F A$ & $9.3 \times 10^{-19}$ & $4.8 \times 10^{-13}$ & Willems et al. 2017 \\
\hline \multicolumn{5}{|c|}{ Bivariate analysis with bone strength index } \\
\hline Appendicular lean mass & FADS1 & & $\mathrm{p}_{\mathrm{b}}=1.6 \times 10^{-07}$ & Han et al. 2012 \\
\hline \multicolumn{5}{|c|}{ Bivariate analysis with appendicular bone size } \\
\hline Appendicular lean mass & GLYAT & & $\mathrm{p}_{\mathrm{b}}=1.8 \times 10^{-06}$ & Guo et al. 2013 \\
\hline
\end{tabular}

$p_{b}$ bivariate $p$ value for bone/muscle pair, $e B M D$ estimated bone mineral density

\section{Shared Biology: Evidence from Multivariate Analysis}

While GWAS are typically performed for the study of one trait at a time, more recently methodological advances have enabled the simultaneous GWAS assessment of multiple traits. In humans, joint analysis of multiple, correlated traits, i.e., multivariate GWAS, has been instrumental to the identification of pleiotropic candidate SNPs/loci associated with traits related to both bone and muscle metabolism. GWAS investigating both bone and muscle phenotypes have produced a list of potential candidate genes for further biological validation such as PRKCH and SCNN1B [30] in 3844 Europeans; HK2, UMOD, MIR873, and MIR876 [31] in 1627 unrelated Chinese adults individuals; HTR1E, COL4A2, AKAP6, SLC2A11, $R Y R 3$, and MEF2C [32]; and GLYAT [33] in 1627 unrelated Chinese adults. In addition, the GWAS-identified METTL21C was found to be a novel pleiotropic gene suggestive of association with both muscle and bone acting through the modulation of the NF- $\mathrm{KB}$ signaling pathway [34••]. This gene has been implicated in the etiology of inclusion body myositis (skeletal muscle) and of early-onset Paget's disease (bone) [35]. Subsequent studies confirmed that METTL21C polymorphisms contribute to peak bone mass in Chinese males [36]. Moreover, frail subjects showed higher expression levels of METT121C compared to young healthy older adults [37]. METTL21C belongs to the METTL21 family of the methyltransferase superfamily and possesses protein-lysine N-methyltransferase activity [38]; its close homolog, METTL21D was found to bind to the chaperone valosin-containing protein
(VCP, a.k.a. VCP/p97), known to play a role in a muscle atrophy disease [39]. More recently, Medina-Gomez et al. [40 • $]$ performed a bivariate GWAS meta-analysis of total body lean mass and total body less head BMD in 10,414 children. The study identified variants with pleiotropic effects in eight loci, mostly already known for BMD (WNT4, GALNT3, MEPE, CPED1/WNT16, TNFSF11, RIN3, and PPP6R3/LRP5), but also the TOM1L2/SREBF1 locus not previously associated with BMD or lean mass. The protein was highly expressed in mouse calvaria-derived cells during osteoblastogenesis and showed the highest expression peak at the onset of osteoblast mineralization in human mesenchymal stem cells. Moreover, SREBP1 indirectly downregulated several key regulators of myogenesis (i.e., MYOD1, MYOG, MEF2C). Notably, SREBF1 exerted opposite effects on the differentiation of myocytes and osteoblasts, which are in line with the opposite effects observed on BMD and lean mass in the bivariate analysis.

\section{Human and Animal Bone-Focused Knock-out "Models" Comprising Muscle Phenotypes}

Congenital disorders affecting bone or muscle are often associated with deficits in the other tissue as well. For example, reduced muscle capacity and strength have been observed in children with osteogenesis imperfecta (OI) [41, 43], where the primary defect comprises the skeletal system. About $85 \%$ of the OI cases are caused by mutations in the COL1A1 and 
COL1A2 genes. These mutations affect the production of the $\alpha 1 / \alpha 2$ chains of type 1 collagen, an important structural component of the bone, skin, tendons, ligaments, and other connective tissues [44]. Animal studies have also observed muscle weakness in OI mice [45], providing additional evidence for the muscle abnormalities in OI. The exact mechanisms leading to muscle weakness are yet unclear but they can be result of intrinsic muscle factors or direct paracrine effects of the abnormal bone matrix (i.e., increased TGF- $\beta$ signaling in OI decreases lean mass).

Further, muscle abnormalities have been also noted in individuals with hypophosphatemic rickets; hereditary phosphate wasting disorders commonly caused by point mutations in PHEX, FGF23, and DMP1 genes. Children carriers of any of these mutations have soft bones (rickets), growth retardation, poor dental development, and elevated serum FGF23 levels [46]. It has been shown that accumulation of FGF23 is strongly associated with muscle function abnormalities in humans [47, 48]. Additionally, murine models have shown reduced grip strength and impaired muscle forces in the Hyp (model of PHEX deficiency) and Dmpl null (DMP1 deficiency) mice [49]. However, the underlying mechanisms leading to skeletal muscle abnormalities in individuals with hypophosphatemic rickets have not been characterized.

\section{Human and Animal Knock-out Models of Muscle Comprising Bone Phenotypes}

Disorders of muscle often present with bone abnormalities. For example, in Duchenne muscular dystrophy (DMD) and Becker muscular dystrophy, the primary defect leading to disease pathogenesis is degeneration of striated muscle. The mutations in the $D M D$ gene encode the dystrophin protein, causing Duchenne and Becker muscular dystrophies. Yet, impairment of bone health in the form of low BMD and increased incidence of bone fractures are well-recognized clinical components of the DMD phenotype [50-52]. The deleterious effects of DMD on bone can be mediated by several mechanisms. These include downstream functional effects on bone involving the nuclear factor of $\mathrm{kB}$ pathway and cytokine-mediated (IL-6) activation of osteopontin (OPN); disturbances of calcium metabolism; deterioration of biomechanical stimuli with disease progression, side effects of corticosteroid treatment and many comorbidity processes derived from the disease. Also, other single-gene disorders including those derived from mutations in MTM1, RYR1, and DNM2 have been implicated in the alteration of skeletal muscles. These congenital myopathies are characterized by an alteration in the contractile apparatus of the muscle (myofibers) followed by loss of muscle mass, increased muscle weakness, and decrease in bone mass. However, due to the complex interplay between muscle and bone, it is not clear if these changes in bone mass are result of the decreased mechanical loading, the muscular paracrine effect, the shared biology, or combination of all of these factors.

Another example of a potentially pleiotropic gene is the myostatin (MSTN, a.k.a. growth and differentiation factor 8, GDF8) gene, a member of the TGF- $\beta$ superfamily, which is secreted primarily by the skeletal muscle cells [53]. Point mutations in MSTN lead to decreased production of functional myostatin causing muscle hypertrophy in humans and animals [54]. Recently, the relationship between myostatin and bone has been actively investigated in animal models. MSTN depletion leads to increased BMD and strength through many different pathways. First and foremost, the loss of myostatin is followed by doubling of the skeletal muscle mass, which increases mechanical loading on bone. However, the increase in lean mass can also have indirect positive effects on the bone by increasing IGF-1 levels [55]. Such studies have found that inhibition of the myostatin pathway increases proliferation and differentiation of osteoprogenitor cells and leads to bone mass accrual [56]. Further, it has been shown that haploinsufficiency of myostatin protects against aging-related declines in muscle function and enhances the longevity of mice [57]. This suggests that, beyond a known effect of myostatin on bone [58], there is also a systemic effect of this gene.

Further, myostatin binds to the soluble activin type IIB receptor (ACVR2B) which forms an activin receptor complex with activin type 1 serine/threonine kinase receptors (ACVRs). Loss of function of activin type I receptor (ACVR1) in osteoblasts increases bone mass and activates canonical Wnt signaling through suppression of the Wnt inhibitors SOST and DKK1 [59••]. Recently, a study has also shown that myostatin inhibits osteoblastic differentiation by suppressing osteocyte-derived exosomal microRNA-218, suggesting a possible novel mechanism in the bone-muscle crosstalk [60].

Myocyte enhancer factor-2 (MEF2C) is a member of the MADS-box superfamily of transcriptional regulatory proteins relevant for skeletal muscle development, sarcomeric gene expression, and fiber type control. MEF2C directly regulates myomesin gene transcription and loss of Mef2c in skeletal muscle results in improper sarcomere organization and disorganized myofibers. Recent studies have found that a super activating form of MEF2C causes precocious chondrocyte hypertrophy, ossification of growth plates, and dwarfism [61]. Mef $2 c^{+/-}$presented lack of ossification within the sternum. Mef2 $c^{\operatorname{loxP} / K O}$; Twist2-Cre mice had shortened limbs from birth [61]. Moreover, the MEF2 activity is enhanced by the increase in mitogen-activated protein kinases (MAPKs) and decrease in histone deacetylases (HDACs). HDAC inhibitors have been tested in a muscular dystrophy model in mice which promoted the formation of muscles with increased cross-sectional area [62]. Interestingly, HDAC5 part of the HDAC family is a known BMD locus [8].

Last but not least, GWAS studies have identified variants in FAM210A as strongly associated with fracture risk but less 
strongly with BMD. Moreover, SNPs near FAM210A were nominally $(p<0.05)$ associated with lean mass in adults [1]. Interestingly, a recent study in mice has shown that Fam $210 a$ was expressed in muscle mitochondria and cytoplasm but not in bone [1]. Notably, grip strength and limb lean mass were reduced in both tamoxifen-inducible Fam210a homozygous global knockout mice (TFam210a $a^{-/-}$) and skeletal muscle cell-specific knockout mice (Fam210aMus ${ }^{-1}$ ). Moreover, microarray analysis showed decreased levels of Myog and Chdh15, transcription factors relevant for myoblast differentiation, and terminal muscle differentiation, respectively. Also, decreased BMD, bone biomechanical strength and bone formation, and elevated osteoclast activity were observed in TFam 210a $a^{-1}$ mice [1]. Furthermore, the authors showed that Mmp12 was increased in muscle cells of TFam210aMus ${ }^{-1}$ mice, which can enhance osteoclast function in bone. Therefore, Fam210a, while being expressed in muscle, plays an influential role on bone quality and quantity.

\section{Muscle and Bone: Beyond Biomechanics}

Multiple metabolic communications have been identified between bone and muscle in humans and rodents. There are numerous indications that the muscle "secretome" contains osteoinducer and osteoinhibitor myokines [63]; it also seems likely that bone cells secrete myoinducer and myoinhibitor osteokines [64]. The skeletal muscle secretome accounts for various molecules that affect bone including insulin-like growth factor-1 (IGF1), fibroblast growth factor (FGF2), interleukins (IL6, IL15), myostatin, osteoglycin, osteoactivin, and others (reviewed by [64]). Even though studies on the potential effects of bone on muscle metabolism are still sparse, a few osteokines have been identified. Prostaglandin E2 (PGE2) and WNT family member 3A (WNT3A), which are secreted by osteocytes, are thought to impact skeletal muscle cells. Interestingly, WNT3A and several other WNT factors have been identified in GWAS of BMD. Also, osteocalcin and IGF-1, which are produced by osteoblasts, and sclerostin, which is secreted by osteocytes and osteoblasts, exert effects on muscle cells. Further, bones and muscles are controlled by mitochondrial genetics that standard GWAS cannot reliably scrutinize given the sparse number of mitochondrial markers on genotyping arrays and the difficulties to quantifying mitochondrial heteroplasmy. Previous studies have revealed the critical role of mitochondria in the differentiation of multiple cell types, including cardiomyocytes [65] and myoblasts [66]. Additionally, osteoporosis and sarcopenia seem to be more prevalent among patients with mitochondrial disorders. Thus, refined GWAS efforts can help understand the underlying mechanisms of the mitochondria, using intensity-based assessments of mitochondrial copy number. In addition to the mitochondrial metabolism, the genes controlling overall energy metabolism might also exert an impact on bone and muscle.
Interestingly, skeletal muscle-specific disruption of the circadian rhythms by Bmall deletion has been shown to disrupt skeletal muscle metabolism [67], whereas BMAL1 deficiency results in a low bone mass phenotype [68]. However, this gene has not yet been identified by any GWAS on BMD.

\section{The Potential of Genetic Discoveries to Guide Drug Target Identification}

Incorporating genetic information in the drug discovery process can improve disease-specific drug target identification and validation. Combining drug mechanisms with genetic mechanistic information increases the success in drug discovery over approaches that do not include genetic information, especially for drug targets related to musculoskeletal (BMD), metabolic, and blood traits [69].

From the molecular factors discussed above, two have been followed as potential drug targets. It has been well established that myostatin is a negative regulator of muscle and bone mass. Therefore, there is hope that studies into myostatin inhibitors may have therapeutic application in treating musclewasting diseases such as muscular dystrophy. Bialek et al. [58] have investigated the role of myostatin by administrating myostatin neutralizing antibody (Mstn-mAb) or soluble myostatin decoy receptor (ActRIIB-Fc) in young adult mice. Interestingly, while both antibodies increased muscle mass, only ActRIIB-Fc also increased bone mass. Thus, a therapeutic agent that has this dual effect represents a potential approach for the simultaneous treatment of osteoporosis and or sarcopenia. Another potential target is FGF23. Currently, clinical trials of neutralizing anti-FGF23 antibody for patients with FGF23-related hypophosphatemic diseases are ongoing. First of all, FGF23 production is stimulated through signaling acting through the FGF receptor. It has also been shown that repeated administration of FGF receptor inhibitors causes increased bone growth and mineralization in Hyp mice [70]. Similarly, weekly injection of FGF23 antibodies increased BMD in Hyp mouse, while with a higher dose, there was also an increase in grip strength. To note, in a phase I clinical trial, administration of various amounts of anti-FGF23 antibodies increased tubular maximum transport of phosphate per glomerular filtration rate (TmP/GFR) in adult patients with $\mathrm{X}$ linked hypophosphatemia (XLH) [71, 72]. Nevertheless, it needs to be tested if anti-FGF23 antibody can improve or cure rickets/osteomalacia or their clinical presentations such as bone pain and muscle weakness. Although the clinical implications of these findings are still far-reaching, both examples illustrate the diverse opportunities for the characterization of drug targets that can prevent muscle and bone abnormalities.

The approach is not free of limitations, as genetically derived targets may also have undesired secondary effects. For example, MEF2C has been suggested as novel drug target for 
therapeutically enhancing muscle performance. Targeting these genes may have a significant impact on the treatment of muscle. However, MEF2C also is related with pathological cardiac hypertrophy. Hence, off-target effects can be considerable. The key will be to improve muscle performance and prevent cardiotoxicity at the same time when targeting these genes [73]. Either way, this also illustrates the potential of the genetic evidence underlying drug targets to typify the presence of adverse effects before embarking on expensive experimentation.

\section{Summary and Future Directions}

Overall, there are many evolutionary, biological, and clinical factors that couple the pathogenesis of sarcopenia and osteoporosis. Both muscle and bone also act as endocrine organs $[74,74]$ and share common genetic influences. Although challenging, there is a growing research enterprise aimed at elucidating and unraveling new mechanisms of muscle-bone crosstalk. Many questions still remain unanswered and need to be addressed through the integration of in vitro and in vivo models. For example, what are the exact mechanisms underlying the cross-organ effects? Do muscle factors, by stimulating bone metabolism, also lead to increased release of myoinducer and myoinhibitor osteokines? More importantly, the question remains as how the aging process influences muscle and bone metabolism, including the underlying molecular factors. Further, the role of central mechanisms in coregulation of the musculoskeletal system needs to be investigated in its entirety rather than its parts. Finally, the study of pleiotropy may provide novel insights into disease pathophysiology with the potential of leading to the identification of drug targets that simultaneously prevent or treat both diseases.

Funding Information D.K. was supported by a Israel Science Foundation grant (No. 1283/14 and 1822/12) and a generous gift from the Samson Family. K.T and F.R are supported by the Netherlands Scientific Organization (NWO) and ZonMW Project number: NWO/ ZONMW-VIDI-0 16-136-367. D.P.K. was supported by NIH grant R01 AR041398 and R01 AR061445.

\section{Compliance with Ethical Standards}

Conflict of Interest Douglas Kiel reports personal fees from Wolters Kluwer for royalties on publication, personal fees from Springer as a book editor, grants from Policy Analysis Inc. (investigator initiated grant to institution on imminent risk of fracture in the Framingham Study), and grants from Dairy Council (Grant to institution on dairy foods and bone health) outside the submitted work.

David Karasik, Katerina Trajanoska, and Fernando Rivadeneira declare no conflict of interest.

Human and Animal Rights and Informed Consent This article does not contain any studies with human or animal subjects performed by any of the authors.
Open Access This article is distributed under the terms of the Creative Commons Attribution 4.0 International License (http:// creativecommons.org/licenses/by/4.0/), which permits unrestricted use, distribution, and reproduction in any medium, provided you give appropriate credit to the original author(s) and the source, provide a link to the Creative Commons license, and indicate if changes were made.

Publisher's Note Springer Nature remains neutral with regard to jurisdictional claims in published maps and institutional affiliations.

\section{References}

Papers of particular interest, published recently, have been highlighted as:

•• Of major importance

[1]•• Tanaka K-I, Xue Y, Nguyen-Yamamoto L, Morris JA, Kanazawa I, Sugimoto T, et al. FAM210A is a novel determinant of bone and muscle structure and strength. Proc Natl Acad Sci U S A. 2018;115:E3759-68. https://doi.org/10.1073/pnas.1719089115 This study have succesfully validated FAM210A as novel gene associated with reduced bone mass and grip strength in genetically modifed mice. FAM210A had been previously discovered to be associated with BMD using GWAS approach.

[2] Reginster J-Y, Beaudart C, Buckinx F, Bruyère O. Osteoporosis and sarcopenia. Curr Opin Clin Nutr Metab Care. 2016;19:31-6. https://doi.org/10.1097/MCO.0000000000000230.

[3] Edwards MH, Dennison EM, Aihie Sayer A, Fielding R, Cooper C. Osteoporosis and sarcopenia in older age. Bone. 2015;80:12630. https://doi.org/10.1016/j.bone.2015.04.016.

[4] Frost HM. Bone's mechanostat: a 2003 update. Anat Rec A Discov Mol Cell Evol Biol. 2003;275:1081-101. https://doi.org/ 10.1002/ar.a.10119.

[5] Avin KG, Bloomfield SA, Gross TS, Warden SJ. Biomechanical aspects of the muscle-bone interaction. Curr Osteoporos Rep. 2015;13:1-8. https://doi.org/10.1007/s11914-014-0244-x.

[6] Verbrugge SAJ, Schönfelder M, Becker L, Yaghoob Nezhad F, Hrabě de Angelis M, Wackerhage H. Genes Whose Gain or Loss-Of-Function Increases Skeletal Muscle Mass in Mice: A Systematic Literature Review. Front Physiol. 2018;9:9. https:// doi.org/10.3389/fphys.2018.00553.

[7] Karasik D, Kiel DP. Genetics of the musculoskeletal system: a pleiotropic approach. J Bone Miner Res. 2008;23:788-802. https://doi.org/10.1359/jbmr.080218.

[8] Estrada K, Styrkarsdottir U, Evangelou E, Hsu Y-H, Duncan EL, Ntzani EE, et al. Genome-wide meta-analysis identifies 56 bone mineral density loci and reveals 14 loci associated with risk of fracture. Nat Genet. 2012;44:491-501. https://doi.org/10.1038/ng.2249.

[9] Duncan EL, Danoy P, Kemp JP, Leo PJ, McCloskey E, Nicholson $\mathrm{GC}$, et al. Genome-wide association study using extreme truncate selection identifies novel genes affecting bone mineral density and fracture risk. PLoS Genet. 2011;7:e1001372. https://doi.org/10. 1371/journal.pgen.1001372.

[10] Rivadeneira F, Styrkársdottir U, Estrada K, Halldórsson BV, Hsu Y-H, Richards JB, et al. Twenty bone-mineral-density loci identified by large-scale meta-analysis of genome-wide association studies. Nat Genet. 2009;41:1199-206. https://doi.org/10.1038/ng.446.

[11] Styrkarsdottir U, Halldorsson BV, Gretarsdottir S, Gudbjartsson DF, Walters GB, Ingvarsson T, et al. Multiple genetic loci for bone mineral density and fractures. N Engl J Med. 2008;358:2355-65. https://doi.org/10.1056/NEJMoa0801197. 
[12] Richards J, Rivadeneira F, Inouye M, Pastinen T, Soranzo N, Wilson S, et al. Bone mineral density, osteoporosis, and osteoporotic fractures: a genome-wide association study. Lancet. 2008;371:1505-12. https://doi.org/10.1016/S0140-6736(08) 60599-1.

[13] Medina-Gomez C, Kemp JP, Trajanoska K, Luan J, Chesi A, Ahluwalia TS, et al. Life-course genome-wide association study meta-analysis of Total body BMD and assessment of age-specific effects. Am J Hum Genet. 2018;102:88-102. https://doi.org/10. 1016/j.ajhg.2017.12.005.

[14] Rivadeneira F, Mäkitie O. Osteoporosis and bone mass disorders: from gene pathways to treatments. Trends Endocrinol Metab. 2016;27:262-81. https://doi.org/10.1016/j.tem.2016.03.006.

[15] Ohlsson C, Henning P, Nilsson KH, Wu J, Gustafsson KL, Sjögren $\mathrm{K}$, et al. Inducible Wnt16 inactivation: WNT16 regulates cortical bone thickness in adult mice. J Endocrinol. 2018;237:113-22. https://doi.org/10.1530/JOE-18-0020.

[16] Movérare-Skrtic S, Wu J, Henning P, Gustafsson KL, Sjögren K, Windahl $\mathrm{SH}$, et al. The bone-sparing effects of estrogen and WNT16 are independent of each other. Proc Natl Acad Sci U S A. 2015;112:14972-7. https://doi.org/10.1073/pnas.1520408112.

[17] Kemp JP, Morris JA, Medina-Gomez C, Forgetta V, Warrington NM, Youlten SE, et al. Identification of 153 new loci associated with heel bone mineral density and functional involvement of GPC6 in osteoporosis. Nat Genet. 2017;49:1468-75. https://doi. org/10.1038/ng.3949.

[18] Morris JA, et al. An atlas of genetic influences on osteoporosis in humans and mice. Nat. Genet. 2018. https://doi.org/10.1038/s41588018-0302-x.

[19] Su J, Ekman C, Oskolkov N, Lahti L, Ström K, Brazma A, et al. A novel atlas of gene expression in human skeletal muscle reveals molecular changes associated with aging. Skelet Muscle. 2015;5: 35. https://doi.org/10.1186/s13395-015-0059-1.

[20] Zillikens MC, Demissie S, Hsu Y-H, Yerges-Armstrong LM, Chou W-C, Stolk L, et al. Large meta-analysis of genome-wide association studies identifies five loci for lean body mass. Nat Commun. 2017;8:80. https://doi.org/10.1038/s41467-017-00031-7.

[21] Chen Z, Wang Z, Lohman T, Heymsfield SB, Outwater E, Nicholas JS, et al. Dual-energy X-ray absorptiometry is a valid tool for assessing skeletal muscle mass in older women. J Nutr. 2007;137:2775-80. https://doi.org/10.1093/jn/137.12.2775.

[22] Karasik D, Zillikens MC, Hsu YH, Aghdassi A, Akesson K, Amin $\mathrm{N}$, et al. Disentangling the genetics of lean mass. Am J Clin Nutr. 2019;109:276-287. https://doi.org/10.1093/ajen/nqy272.

[23] Willems SM, Wright DJ, Day FR, Trajanoska K, Joshi PK, Morris JA, et al. Large-scale GWAS identifies multiple loci for hand grip strength providing biological insights into muscular fitness. Nat Commun. 2017;8:16015.

[24] Tikkanen E, Gustafsson S, Amar D, Shcherbina A, Waggott D, Ashley EA, et al. Biological insights into muscular strength: genetic findings in the UK biobank. Sci Rep. 2018;8:6451. https:// doi.org/10.1038/s41598-018-24735-y.

[25] Sonnemann KJ, Fitzsimons DP, Patel JR, Liu Y, Schneider MF, Moss RL, et al. Cytoplasmic $\gamma$-actin is not required for skeletal muscle development but its absence leads to a progressive myopathy. Dev Cell. 2006;11:387-97. https://doi.org/10.1016/j.devcel. 2006.07.001.

[26] Burr AR, Millay DP, Goonasekera SA, Park KH, Sargent MA, Collins $\mathrm{J}$, et al. $\mathrm{Na}+$ dysregulation coupled with $\mathrm{Ca} 2+$ entry through $\mathrm{NCX} 1$ promotes muscular dystrophy in mice. Mol Cell Biol. 2014;34:19912002. https://doi.org/10.1128/MCB.00339-14.

[27] Dale JM, Shen H, Barry DM, Garcia VB, Rose FF, Lorson CL, et al. The spinal muscular atrophy mouse model, SMA $\Delta 7$, displays altered axonal transport without global neurofilament alterations. Acta Neuropathol. 2011;122:331-41. https://doi.org/10. 1007/s00401-011-0848-5.
[28] Paaby AB, Rockman MV. The many faces of pleiotropy. Trends Genet. 2013;29:66-73. https://doi.org/10.1016/j.tig.2012.10.010.

[29] Solovieff N, Cotsapas C, Lee PH, Purcell SM, Smoller JW. Pleiotropy in complex traits: challenges and strategies. Nat Rev Genet. 2013;14:483-95. https://doi.org/10.1038/nrg3461.

[30] Gupta M, Cheung C-L, Hsu Y-H, Demissie S, Cupples LA, Kiel DP, et al. Identification of homogeneous genetic architecture of multiple genetically correlated traits by block clustering of genome-wide associations. J Bone Miner Res. 2011;26:1261-71. https://doi.org/10.1002/jbmr.333.

[31] Sun L, Tan L-J, Lei S-F, Chen X-D, Li X, Pan R, et al. Bivariate genome-wide association analyses of femoral neck bone geometry and appendicular lean mass. PLoS One. 2011;6:e27325. https:// doi.org/10.1371/journal.pone.0027325.

[32] Karasik D, Cheung CL, Zhou Y, Cupples LA, Kiel DP, Demissie $\mathrm{S}$. Genome-wide association of an integrated osteoporosis-related phenotype: is there evidence for pleiotropic genes? J Bone Miner Res. 2012;27:319-30. https://doi.org/10.1002/jbmr.563.

[33] Guo Y-F, Zhang L-S, Liu Y-J, Hu H-G, Li J, Tian Q, et al. Suggestion of GLYAT gene underlying variation of bone size and body lean mass as revealed by a bivariate genome-wide association study. Hum Genet. 2013;132:189-99. https://doi.org/10. 1007/s00439-012-1236-5.

[34]•• Huang J, Hsu Y-H, Mo C, Abreu E, Kiel DP, Bonewald LF, et al. METTL21C Is a Potential Pleiotropic Gene for Osteoporosis and Sarcopenia Acting Through the Modulation of the NF-kB Signaling Pathway. J Bone Miner Res. 2014;29:1531-40. https:// doi.org/10.1002/jbmr.2200 This study showed that METTL21C plays important role in mygenesis and osteocyte homeostasis via the $\mathrm{NF} \kappa \mathrm{B}$ signaling pathway using $\mathrm{C2C12}$ myoblasts/ myotubes and MLO-Y4 osteocyte-like cells.

[35] Cloutier P, Lavallée-Adam M, Faubert D, Blanchette M, Coulombe B. A newly uncovered Group of Distantly Related Lysine Methyltransferases Preferentially Interact with molecular chaperones to regulate their activity. PLoS Genet. 2013;9: e1003210. https://doi.org/10.1371/journal.pgen.1003210.

[36] Zhao F, Gao L, Li S, Wei Z, Fu W, He J, et al. Association between SNPs and haplotypes in the METTL21C gene and peak bone mineral density and body composition in Chinese male nuclear families. J Bone Miner Metab. 2017;35:437-47. https://doi.org/ 10.1007/s00774-016-0774-7.

[37] Hangelbroek RWJ, Fazelzadeh P, Tieland M, Boekschoten MV, Hooiveld GJEJ, van Duynhoven JPM, et al. Expression of protocadherin gamma in skeletal muscle tissue is associated with age and muscle weakness. J Cachexia Sarcopenia Muscle. 2016;7: 604-14. https://doi.org/10.1002/jcsm.12099.

[38] Kernstock S, Davydova E, Jakobsson M, Moen A, Pettersen S, Mælandsmo GM, et al. Lysine methylation of VCP by a member of a novel human protein methyltransferase family. Nat Commun. 2012;3:1038. https://doi.org/10.1038/ncomms2041.

[39] Cohen S, Nathan JA, Goldberg AL. Muscle wasting in disease: molecular mechanisms and promising therapies. Nat Rev Drug Discov. 2015;14:58-74. https://doi.org/10.1038/nrd4467.

[40]•• Medina-Gomez C, Kemp JP, Dimou NL, Kreiner E, Chesi A, Zemel $\mathrm{BS}$, et al. Bivariate genome-wide association meta-analysis of pediatric musculoskeletal traits reveals pleiotropic effects at the SREBF1/ TOM1L2 locus. Nat Commun. 2017;8:121. https://doi.org/10.1038/ s41467-017-00108-3 Using a bivaraite GWAS approach, this study have found a novel gene with pleiotropic effects on bone and muscle, which was expressed in murine and human osteoblasts, as well as in human muscle tissue.

[41] Takken T, Terlingen HC, Helders PJM, Pruijs H, van Der Ent CK, Engelbert RHH. Cardiopulmonary fitness and muscle strength in patients with osteogenesis imperfecta type I. J Pediatr. 2004;145: 813-8. https://doi.org/10.1016/j.jpeds.2004.08.003. 
[42] Caudill A, Flanagan A, Hassani S, Graf A, Bajorunaite R, Harris G, et al. Ankle strength and functional limitations in children and adolescents with type I osteogenesis imperfecta. Pediatr Phys Ther. 2010;22: 288-95. https://doi.org/10.1097/PEP.0b013e3181ea8b8d.

[43] Pokidysheva E, Mizuno K, Bächinger HP. The collagen folding machinery: biosynthesis and post-translational modifications of collagens. Osteogenes Imperfecta 2014;57-70. doi:https://doi. org/10.1016/B978-0-12-397165-4.00006-X.

[44] Gentry BA, Ferreira JA, McCambridge AJ, Brown M, Phillips CL. Skeletal muscle weakness in osteogenesis imperfecta mice. Matrix Biol. 2010;29:638-44. https://doi.org/10.1016/j.matbio.2010.06. 006.

[45] Guven A, Al-Rijjal RA, BinEssa HA, Dogan D, Kor Y, Zou M, et al. Mutational analysis of PHEX, FGF23 and CLCN5 in patients with hypophosphataemic rickets. Clin Endocrinol. 2017;87: 103-12. https://doi.org/10.1111/cen.13347.

[46] Veilleux L-N, Cheung M, Ben Amor M, Rauch F. Abnormalities in muscle density and muscle function in Hypophosphatemic rickets. J Clin Endocrinol Metab. 2012;97:E1492-8. https://doi.org/ 10.1210/jc.2012-1336.

[47] Veilleux L-N, Cheung MS, Glorieux FH, Rauch F. The musclebone relationship in X-linked Hypophosphatemic rickets. J Clin Endocrinol Metab. 2013;98:E990-5. https://doi.org/10.1210/jc. 2012-4146.

[48] Wacker MJ, Touchberry CD, Silswal N, Brotto L, Elmore CJ, Bonewald LF, et al. Skeletal muscle, but not cardiovascular function, Is Altered in a Mouse Model of Autosomal Recessive Hypophosphatemic Rickets. Front Physiol. 2016;7:173. https:// doi.org/10.3389/fphys.2016.00173.

[49] Ma J, McMillan HJ, Karagüzel G, Goodin C, Wasson J, Matzinger MA, et al. The time to and determinants of first fractures in boys with Duchenne muscular dystrophy. Osteoporos Int. 2017;28: 597-608. https://doi.org/10.1007/s00198-016-3774-5.

[50] Trinh A, Wong P, Brown J, Hennel S, Ebeling PR, Fuller PJ, et al. Fractures in spina bifida from childhood to young adulthood. Osteoporos Int. 2017;28:399-406. https://doi.org/10.1007/ s00198-016-3742-0.

[51] Mughal MZ. Fractures in children with cerebral palsy. Curr Osteoporos Rep. 2014;12:313-8. https://doi.org/10.1007/s11914014-0224-1.

[52] Hamrick MW. The skeletal muscle secretome: an emerging player in muscle-bone crosstalk. Bonekey Rep. 2012;1:60. https://doi. org/10.1038/bonekey.2012.60.

[53] Mosher DS, Quignon P, Bustamante CD, Sutter NB, Mellersh CS, Parker HG, et al. A mutation in the Myostatin gene increases muscle mass and enhances racing performance in heterozygote dogs. PLoS Genet. 2007;3:e79. https://doi.org/10.1371/journal. pgen.0030079.

[54] Williams NG, Interlichia JP, Jackson MF, Hwang D, Cohen P, Rodgers BD. Endocrine actions of Myostatin: systemic regulation of the IGF and IGF binding protein Axis. Endocrinology. 2011;152:172-80. https://doi.org/10.1210/en.2010-0488.

[55] Elkasrawy MN, Hamrick MW. Myostatin (GDF-8) as a key factor linking muscle mass and bone structure. J Musculoskelet Neuronal Interact. 2010;10:56-63.

[56] Mendias CL, Bakhurin KI, Gumucio JP, Shallal-Ayzin MV, Davis CS, Faulkner JA. Haploinsufficiency of myostatin protects against aging-related declines in muscle function and enhances the longevity of mice. Aging Cell. 2015;14:704-6. https://doi.org/10. 1111/acel.12339.

[57] Bialek P, Parkington J, Li X, Gavin D, Wallace C, Zhang J, et al. A myostatin and activin decoy receptor enhances bone formation in mice. Bone. 2014;60:162-71. https://doi.org/10.1016/j.bone. 2013.12.002.

[58] Kamiya N, Kaartinen VM, Mishina Y. Loss-of-function of ACVR1 in osteoblasts increases bone mass and activates canonical Wnt signaling through suppression of Wnt inhibitors SOST and DKK1. Biochem Biophys Res Commun. 2011;414: 326-30. https://doi.org/10.1016/j.bbrc.2011.09.060.

[59]•• Qin Y, Peng Y, Zhao W, Pan J, Ksiezak-Reding H, Cardozo C, et al. Myostatin inhibits osteoblastic differentiation by suppressing osteocyte-derived exosomal microRNA-218: A novel mechanism in muscle-bone communication. J Biol Chem. 2017;292:1102133. https://doi.org/10.1074/jbc.M116.770941 This study reported that myostatin promotes expression of several bone regulators such as SOST, DKK1, and RANKL in cultured osteocytic cells, which in turn exerts an inhibitory effect on osteoblast differentiation.

[60] Arnold MA, Kim Y, Czubryt MP, Phan D, McAnally J, Qi X, et al. MEF2C transcription factor controls chondrocyte hypertrophy and bone development. Dev Cell. 2007;12:377-89. https://doi.org/10. 1016/j.devcel.2007.02.004.

[61] Consalvi S, Mozzetta C, Bettica P, Germani M, Fiorentini F, Del Bene F, et al. Preclinical studies in the mdx mouse model of duchenne muscular dystrophy with the histone deacetylase inhibitor givinostat. Mol Med. 2013;19:79-87. https://doi.org/10.2119/ molmed.2013.00011.

[62] Pedersen BK, Febbraio MA. Muscles, exercise and obesity: skeletal muscle as a secretory organ. Nat Rev Endocrinol. 2012;8:45765. https://doi.org/10.1038/nrendo.2012.49.

[63] Tagliaferri C, Wittrant Y, Davicco M-J, Walrand S, Coxam V. Muscle and bone, two interconnected tissues. Ageing Res Rev. 2015;21:55-70. https://doi.org/10.1016/j.arr.2015.03.002.

[64] Chung S, Dzeja PP, Faustino RS, Perez-Terzic C, Behfar A, Terzic A. Mitochondrial oxidative metabolism is required for the cardiac differentiation of stem cells. Nat Clin Pract Cardiovasc Med. 2007;4:S60-7. https://doi.org/10.1038/ncpcardio0766.

[65] Rochard P, Rodier A, Casas F, Cassar-Malek I, Marchal-Victorion $\mathrm{S}$, Daury L, et al. Mitochondrial activity is involved in the regulation of myoblast differentiation through myogenin expression and activity of myogenic factors. J Biol Chem. 2000;275:2733-44.

[66] Ehlen JC, Brager AJ, Baggs J, Pinckney L, Gray CL, DeBruyne JP, et al. Bmall function in skeletal muscle regulates sleep. Elife 2017;6. doi:https://doi.org/10.7554/eLife.26557.

[67] Samsa WE, Vasanji A, Midura RJ, Kondratov RV. Deficiency of circadian clock protein BMAL1 in mice results in a low bone mass phenotype. Bone. 2016;84:194-203. https://doi.org/10.1016/j. bone.2016.01.006.

[68] Nelson MR, Tipney H, Painter JL, Shen J, Nicoletti P, Shen Y, et al. The support of human genetic evidence for approved drug indications. Nat Genet. 2015;47:856-60. https://doi.org/10.1038/ ng.3314.

[69] Wöhrle S, Henninger C, Bonny O, Thuery A, Beluch N, Hynes NE, et al. Pharmacological inhibition of fibroblast growth factor (FGF) receptor signaling ameliorates FGF23-mediated hypophosphatemic rickets. J Bone Miner Res. 2013;28:899-911. https://doi.org/10.1002/jbmr.1810.

[70] Carpenter TO, Imel EA, Ruppe MD, Weber TJ, Klausner MA, Wooddell MM, et al. Randomized trial of the anti-FGF23 antibody KRN23 in X-linked hypophosphatemia. J Clin Invest. 2014;124: 1587-97. https://doi.org/10.1172/JCI72829.

[71] Imel EA, Zhang X, Ruppe MD, Weber TJ, Klausner MA, Ito T, et al. Prolonged correction of serum phosphorus in adults with Xlinked hypophosphatemia using monthly doses of KRN23. J Clin Endocrinol Metab. 2015;100:2565-73. https://doi.org/10.1210/jc. 2015-1551.

[72] Potthoff MJ, Wu H, Arnold MA, Shelton JM, Backs J, McAnally $\mathrm{J}$, et al. Histone deacetylase degradation andMEF2 activation promote the formation of slow-twitch myofibers. J Clin Invest. 2007;117:2459-67. https://doi.org/10.1172/JCI31960.

[73] Elefteriou F, Ahn JD, Takeda S, Starbuck M, Yang X, Liu X, et al. Leptin regulation of bone resorption by the sympathetic nervous 
system and CART. Nature. 2005;434:514-20. https://doi.org/10. 1038/nature03398.
[74] Oury F, Sumara G, Sumara O, Ferron M, Chang H, Smith CE, et al. Endocrine regulation of male fertility by the skeleton. Cell. 2011;144:796-809. https://doi.org/10.1016/j.cell.2011.02.004. 